\title{
RI0. CHIKUNGUNYA VIRUS (CHIKV) OUTBREAK IN FEIRA DE SANTANA, BAHIA: PRELIMINARY REPORT.
}

Karen Trinta ${ }^{1}$; Marisa Ribeiro ${ }^{2}$; Marcela Maurell2; Michel Sucupira ${ }^{2}$; Camila Montalbano; ${ }^{3}$ Maricélia Maia ${ }^{4}$; Márcia Dal Fabbro5; Rivaldo Venâncio da Cunha ${ }^{6}$.

${ }^{1}$ LATED / Bio-Manguinhos / Fiocruz;

${ }^{2}$ LATED / Bio-Manguinhos / Fiocruz;

${ }^{3}$ Universidade Federal do Mato Grosso do Sul (UFMS);

${ }^{4}$ Secretaria de Vigilância Sanitária de Feira de Santana, BA;

${ }^{5}$ Secretaria de Saúde de Campo Grande, MS;

${ }^{6}$ Fiocruz - MS.

INTRODUCTION CHIKV is an arthropod-borne virus that is transmitted by Aedes mosquitoes. The infection is characterized by painful arthralgia that may last over two years after the onset of the illness. The first autochthonous cases of CHIKV infection in Brazil were reported in Oiapoque - AM, on September 2014. On the same month, autochthonous cases were also confirmed in Feira de Santana - BA. By February 2016,26.952 cases were reported in 14 Brazilian states. The patient's disability during CHIKV infection is a serious public health problem, which can economically affect the country's health system.

OBJECTIVE The aim of this study is to evaluate clinical, epidemiological and laboratory aspects of Chikungunya virus infection in Feira de Santana, BA. The samples obtained in this study will also be used for the assembly of serological panels for validation of diagnostic tests that are being developed in Bio-Manguinhos.

METHODOLOGY Serial samples from patients with clinical symptoms of CHIKV infection, spanning an observation period of six months, are being analyzed. All patients filled out a detailed questionnaire in order to collect epidemiological data. Serum samples are being tested for IgM and IgG antibodies against CHIKV and Dengue virus, using an enzyme linked immunosorbent assay (ELISA) employing recombinant antigens. They are also being tested for the presence of Chikungunya, Dengue and Zika virus RNA, by a real time RT-PCR.

RESULTS Until now, 188 patients with clinical symptoms of CHIKV infection were enrolled in this study. A total of 575 samples were obtained. So far, 116 serum 118 
samples obtained from the first visit to Feira de Santana, have been tested for IgM anti-CHIKV, 58 were positive (50\%). One hundred and thirty five samples were tested by real time RT-PCR for the presence of CHIKV and Dengue RNA, 47 were positive (34.2\%) for CHIKV. None of the samples were positive for Dengue virus RNA. Twenty nine samples suspect of Zika virus infection were tested by real time RT-PCR, six were positive (21.7\%). The initial analysis of the questionnaires has shown cases of chronic joint pain in a high number of patients infected with CHIKV.

CONCLUSION We are still working on samples characterization and data analyzes, however, the initial data showed a high frequency of CHIKV infection in the studied population, as well as Zika virus circulation. With our results we expect to obtain a well-characterized panel of positive samples for CHIKV and also Zika virus that can be used by Biomanguinhos for validation of serological tests.

KEYWORDS Chikungunya virus, epidemiology, Feira de Santana. 